Article

\title{
Non-equilibrium Thermodynamic Foundations of the Origin of Life
}

\author{
Karo Michaelian \\ Department of Nuclear Physics and Application of Radiation, Instituto de Física, Universidad Nacional \\ Autónoma de México, Circuito Interior de la Investigación Científica, Cuidad Universitaria, Cuidad de \\ México, C.P. 04510.; karo@fisica.unam.mx
}

Version January 30, 2022 submitted to Journal Not Specified

\begin{abstract}
There is little doubt that life's origin followed from the known physical and chemical laws of Nature. The most general scientific framework incorporating the laws of Nature and applicable to most known processes to good approximation, is that of thermodynamics and its extensions to treat out-of-equilibrium phenomena. The event of the origin of life should therefore also be amenable to such an analysis. In this paper, I describe the non-equilibrium thermodynamic foundations of the origin of life for the non-expert. This "Thermodynamic Dissipation Theory for the Origin of Life" is founded on Classical Irreversible Thermodynamic theory developed by Lars Onsager, Ilya Prigogine, and coworkers.
\end{abstract}

Keywords: origin of life; disspative structuring; prebiotic chemistry; abiogenisis; non-equilibrium thermodynamics; thermodynamic dissipation theory

MSC: 92C05, 92C15, 92C $40,92 \mathrm{C} 45,80 \mathrm{Axx}, 82 \mathrm{Cxx}, 82 \mathrm{~B} 35,82 \mathrm{C} 26$

\section{Introduction}

Theories concerning the origin of life on Earth began with captivating myths, prevalent in all cultures, describing how the heavens, Earth, and life were created by powerful supernatural beings. The ancient Greeks were the first of the cultures to leave a written history suggesting a materialistic origin, arguing that life evolved out of the essential elements; water, earth, air, and fire. Over the last 2000 years, however, most of humanity has preferred mysticism over materialism and has accepted the monotheistic Judaeo-Christian-Islamic conception of an omnipotent supernatural being who breathed life into clay models.

With the European Renaissance beginning in the $15^{\text {th }}$ century came a re-invigoration of the art of inquiry and observation, known as "science", first championed by the ancient Greeks. Materialistic ideas of the origin of life once again became popular, for example, the re-adoption of the ancient Greek idea that something as developed as a worm, a fly, or a frog could emerge from mud, dirty clothes, or a puddle of dirty water; the idea of "spontaneous generation" which remained prevalent until the middle of the 19th century.

The last 100 years or so, has seen legends, myths and spurious creation theories give way to more scientific attempts to explain the origin of life. The new materialistic theories, based on a more profound understanding of physics and chemistry, suggested, for example, the possibility of life emerging from sets of auto-catalytic chemical reactions [1,2] occurring perhaps in particular nutrient and energy rich environments such as at hydro-thermal vents at the bottom of the ocean [3], or in cyclical wetting and drying periods on clay mineral surfaces [4].

Even these currently popular, materialistic, and more science based theories for the origin of life, however, lack axiomatic foundations based in physical law and instead settle for describing only the 
chemical synthesis of as many fundamental molecules of life (common to all three life domains; archea, bacteria, eukaryote) as possible, as efficiently as possible, and from an as limited set of precursor molecules as possible. All such theories, however, share a similar characteristic of their mystical counterparts; they rely on the unsettling premise that Nature experienced an apparently unique, almost miraculous, chemical reaction event 3.9 billion years ago, endowing these molecules with Darwinian like characteristics of reproduction with variation and selection based on either efficacy of molecular precursor sequestration or molecular stability. Suffice to say that no such miraculous chemical reaction set has yet been discovered.

How the fundamental molecules of life could have been synthesized from simpler common precursor molecules, before the existence of the complex bio-synthetic pathways, is considered basically a solved problem in origin of life research. In fact, many different chemical and photochemical routes, under different chemical/physical environments, to the production of these molecules, from what would have been commonly available precursor molecules, have been discovered over the past 70 years of experimentation since the first results of Stanley Miller.

Life, however, is much more than a simple collection of fundamental molecules arranged in a particular pattern. This can be seen most clearly from the fact that a recently dead organism contains all of the molecules and their arrangement of a living organism, but there is an obvious difference between a recently dead animal and one which is alive, displaying a certain living spirit or vitality to it. The collection of molecules in the living organism manifests macroscopic dynamical processes such as; mobility, metabolism, homeostasis, replication/proliferation, and the ability to evolve and adapt to different environments. Clearly, there is something more to be explained concerning the origin of life than the mere description of efficient chemical reaction routes to the fundamental molecules.

To many scientists this vitality was so confounding that it seemed obvious to them that the explanation of life required the discovery of some new law of Nature. The eminent physicist Eugene Wigner wrote in the 1970's, [5],

...the laws of physics, applicable for inanimate matter, will have to be modified when dealing with the more general situation in which life and consciousness play significant roles.

This problem concerning the vitality of life has been more difficult to solve than the molecular production problem, but its contemplation is hardly new. Although only in the last few decades have scientists begun to focus on this vitality, part of the understanding had already been forged and was being referenced to sporadically, beginning 150 years ago by the same erudite physicist who provided a statistical mechanical foundation for the empirical science of thermodynamics, Ludwig Boltzmann. Reflecting deeply on living systems and the confounding aspects of Darwin's novel perspective on biological evolution with its "struggle for existence", Boltzmann in 1886 wrote in an essay [6,7],

The general struggle for existence of animate beings is not a struggle for raw materials these, for organisms, are air, water and soil, all abundantly available - nor for energy which exists in plenty in any body in the form of heat, but a struggle for [negative] entropy, which becomes available through the transition of energy from the hot sun to the cold earth.

Boltzmann saw the process of life as a struggle of organisms to obtain and maintain their organization, and his analysis showed that this could only come at the expense of disorganization in the environment. In particular, Boltzmann realized that the organization of living organisms is allowed by the disorganization of the energy given off by the sun and received by Earth. The words organization and disorganization are used loosely here to refer to the probability distribution of certain conserved quantities of Nature inherent to material; energy, momentum, angular momentum, and charge, over microscopic degrees of freedom of the material. The "microscopic degrees of freedom" refer to ways of storing energy at the microscopic level (for example, molecular translation, rotation, and vibration). When these conserved quantities are distributed over fewer degrees of freedom, we say that the system is "organized" and has low entropy (for example the kinetic energy of a hammer 
before it hits a piece of metal), and when distributed over many degrees of freedom, we say the system is "disorganized" and has greater entropy (after the hammer crashes into the metal its kinetic energy of motion is converted into heat - you can actually do the experiment and feel the metal heat up). What we call the "entropy increase" for this event is a measure of the greater distribution of the initial hammer kinetic energy over many microscopic degrees of freedom which correspond to the atomic vibrations and electron motions in the metal, and eventually over the microscopic degrees of freedom of the environment, the surrounding air and ground.

The importance of Boltzmann's insight will be discussed in section 2, however, suffice to say here that Boltzmann showed that the ordering of material in space and time (what we call dynamical processes) could only happen together with concomitant greater disorganization of material or energy in another connected (causal) space-time region, and this region is given the name "the environment".

Ilya Prigogine, building on the work of Boltzmann and his mentor Lars Onsager, showed that under an imposed general force (for example a temperature gradient, a chemical potential, or a photon flux) the material in the system could "self-organize" into dissipative structures which are "designed" by Nature to increase the dissipation of the imposed general force. Examples of dissipative structures are, hurricanes, convection cells, and a set of chemical reactions. A hurricane, for example, is "designed" by Nature to spread the greater energy in the hot ocean surface over the cold upper atmosphere, leading to a more probable distribution of the energy over all the available microscopic degrees of freedom, effectively attempting to reduce the temperature gradient between the ocean and atmosphere. These dissipative structures are created under the impressed general force as a result of the thermodynamic imperative of dissipation, which derives from the local formulation of the Second Law of thermodynamics [8].

It will be shown in this paper that the relevant conserved quantity involved in the dissipative structuring at the origin of life is energy (incident photon energy), and that the relevant microscopic degrees of freedom involved in the dissipation of this energy are molecular and involve electronic excitation, molecular vibration and reconfiguration (e.g. new covalent bonding between atoms), leading to heat (molecular vibrations giving rise to photon emission at longer wavelengths). These material degrees of freedom are places where the low entropy incident energy is absorbed and redistributed and then re-emitted as higher entropy radiation to the environment.

The Thermodynamic Dissipation Theory of the Origin and Evolution of Life postulates that the first molecules of life (the fundamental molecules) were, at their origin, pigments dissipatively structured on the surface of the oceans from simpler and more common precursor molecules in water under the solar UVC light of the Archean. They were "designed" by Nature to carry out this thermodynamic imperative of absorbing light in the UVC region and then to dissipate it into heat (longer wavelength photons) released into the environment. This paper is dedicated to explaining how, and why, this happens in detail.

If the thermodynamic dissipation theory for the origin of life proves to be the correct theory, then, given particular, but probably common, environmental conditions on planets of hot K-type, G-type, and hotter stars (having an important UVC component), there will be, in fact, a thermodynamic imperative for an origin of life similar to our own based on carbon. In this case, the origin of life would not have been a single fortuitous chemical event constrained to perhaps a few "lucky" planets, but rather a thermodynamic imperative and would appear on many, if not most planets, of these types of stars. In this case we would certainly not be alone in the universe, although, as we will see, because of the specific physical requirements for carbon based molecular dissipative structuring (e.g. UVC light limited to wavelengths greater than $205 \mathrm{~nm}$, high temperature, neutral atmosphere, neutral solvent $\mathrm{pH}$, etc.), developed complex lifeforms based on carbon, such as we know them on Earth, may be relatively scarce. However, the first steps of life, which our theory predicts would correspond to the "self-organization" of organic pigments, should be occurring on the surface of every planet circling any star which has at least some continuous light in the 205 to $285 \mathrm{~nm}$ UVC region and shielded by an 
atmosphere from wavelengths shorter than about $205 \mathrm{~nm}$ which can sequentially ionize carbon based pigments and thereby destroy them.

We therefore begin with the not so unreasonable proposition that the fundamental molecules of life, including the nucleobases and their stacking into DNA and RNA polymers, were, at life's origin, "self-organized" dissipative structures arising under the UVC photons that reached Earth's surface at the beginning of the Archean. The general thermodynamic considerations for dissipative structuring will be given in the following section. A description of the thermodynamic dissipation theory for the origin of life, and evidence for it, will be given in section 3. Exactly how the dissipative structuring under UVC light of HCN in water leads to the nucleobases will be described in section 3.2. In section 3.3 I show how a related phenomena, which we call dissipative proliferation, arises from the autocatalytic property of these molecules in promoting themselves under this UVC light and results in their proliferation over the whole surface of Earth. In section 3.5 entitled "Ultarviolet and Temperature Assisted Replication (UVTAR)" I describe how the dissipation of UVC light within these nucleobases drove the denaturing of DNA and RNA polymers during the afternoon, thus allowing their template directed reproduction overnight with the aid of transition metal ion catalysts. In section 3.6 I describe how the association between DNA or RNA codons (or anticodons) and their cognate amino acids formed through dissipative structuring because this led to increased photon dissipation for the DNA/RNA-amino acid complex. Finally, in section 3.7, I describe how homochirality of the fundamental molecules would have arisen naturally if the UVTAR mechanism for denaturing RNA and DNA was operating asymmetrically (morning/afternoon) on the Archean ocean surface.

\section{General Thermodynamic Considerations}

With the development of equilibrium thermodynamics beginning around the middle of the $19^{\text {th }}$ century, and its successful application to improving the efficiencies of the new machines of the industrial revolution, it was imagined that life could also be treated under such a formalism. At first, though, it seemed that life contradicted the new thermodynamic theory since isolated systems would inevitably evolve towards the equilibrium state, as directed by the Second Law of thermodynamics, in which the conserved quantities would be maximally distributed over the microscopic degrees of freedom (maximum entropy) thereby excluding any macroscopic organization in space and time (vitality or dynamics).

This apparent puzzle persists even today, especially among those who prefer that the origin of life remain a mystery inaccessible to human comprehension, and thereby best ascribable to a omnipotent supernatural being. Many non-specialists also incorrectly assume that the second law is applicable to all systems, life included. However, Boltzmann, even in 1886, realized that systems can be either isolated (no flow of energy or matter, or work performed, over the boundary of the system), closed (only energy flow and work performed over the boundary), or open (open to a flow of energy, mass, and work) and that the Second Law is only valid for isolated systems. Life is obviously not an isolated system, but rather an open system, exchanging matter and energy with its environment. Living systems, therefore, are not subject to the Second Law.

But then, what about open systems like life? Was there anything from thermodynamic theory that could be used to predict the evolution of an open system? The problem wasn't completely resolved until the mid 1950's when Prigogine, Glansdorff, Nicolis, and others, based on original work of Lars Onsager (Nobel Prize Chemistry 1968), developed the mathematical framework known as Classical Irreversible Thermodynamics [8] (non-equilibrium thermodynamics) to treat systems open to their environment and affected by the flow of entropy attached to the flow of material or energy coming into the system from the external environment. These same researchers also realized that non-linear phenomena (i.e. a flow non-linearly proportional to the imposed force causing it) were more of a rule than an exception in natural systems and that this non-linearity enabled a whole new paradigm of dynamics (or vitality) for these open systems. 
Especially important in the analysis of Prigogine and coworkers was how the non-linearity, between flows of material and energy and their corresponding forces, gave rise to the possibility of multiple dynamical meta-stable states for a system, including states in which there appeared to be a spontaneous symmetry breaking and associated structuring of material in space and time within the system. As Boltzmann had first observed, such a structured system had the particular property of inevitably increasing the rate of the spread of the conserved quantities (energy, momentum, angular momentum, charge) over the microscopic degrees of freedom of the environment, or, in other words, increasing the rate of dissipation of the imposed external force (rate of entropy production). Prigogine called these structures dissipative structures and showed that they arise only far from equilibrium, where large external forces took the system into the non-linear regime.

The realization that non-linear systems had multiple dynamical meta-stable states, unlike the one static equilibrium state, meant that the system, responding to perturbation, could evolve over these states, and the direction of evolution for many biological systems tended towards states with greater entropy production (greater rate of distribution of the conserved quantities of nature over more microscopic degrees of freedom). A deep understanding of many complex phenomena in Nature, including meteorological systems, turbulence, and the vitality of life, thus began to emerge.

In summary, isolated systems always moved towards the equilibrium state, of which there is, always, only one, and this state corresponds to a maximum of entropy, i.e. to a maximum probability distribution of the conserved quantities over all the microscopic degrees of freedom. No enduring macroscopic dynamics or vitality is allowed for isolated systems, they inevitably decay towards a static equilibrium state since any dynamics would imply that the conserved quantity of energy or momentum was not distributed with maximal probability over all microscopic degrees of freedom. Open systems like life, or even closed systems (only work and energy flow over the system, but no mass flow) could, however, evolve to dynamical meta-stable states of which there could be many, and the system could evolve from one such state to another depending on its initial conditions and subsequent perturbations.

In the process of this evolution, the open system could become more organized (structuring not only in space, but also in time, implying a macroscopic process with dynamic vitality). Even more interesting, this structuring, in the case of non-linear systems with positive feedback, provided greater efficacy for the distribution of the conserved quantities over more microscopic degrees of freedom of the environment, i.e. greater entropy production rates. For this reason, these structures were given the name "dissipative structures" by Prigogine. We are already familiar with many of the largest macroscopic of these dynamical dissipative structures on Earth, for example, hurricanes, tornadoes, convection cells, the water cycle, global trade winds, ocean currents, etc.

Scientists have also, more recently, become familiar with microscopic dissipative structures, such as, for example the molecular machines and the different organelles (factories) operating within a living cell.

A comprehensive theory for the origin of life, based on firmly established physical principles, thus became possible after Prigogine's work of the 1950's and 1960's on dissipative structuring, but the specific details of how the dissipative structuring of life actually arose were only revealed beginning with our analysis around $2008[9,10]$. We realized that the fundamental molecules of life could themselves have been self-organized microscopic dissipative structures, which arose under the thermodynamic imperative to increase dissipation of the incident photons. In particular, we suggested that the fundamental molecules were dissipatively structured as pigments to convert the UVC solar light available at Earth's surface during the Archean into heat (i.e. a greater distribution of the absorbed incident energy over a greater number of emitted photons). We called this theory the Thermodynamic Dissipation Theory for the Origin and Evolution of Life and will now only briefly describe it since detailed descriptions of all aspects of the theory can be found elsewhere [9-24]. 


\section{The Thermodynamic Dissipation Theory of the Origin of Life}

The proposed thermodynamic dissipation theory suggests that life arose as dissipative structuring of UVC pigments on the surface of Earth's oceans to augment the global entropy production through photon dissipation. In fact, any dissipative structure, also known as an "irreversible process," originates and persists for only this fundamental reason; to distribute the conserved quantities of nature over ever more microscopic degrees of freedom, i.e. to produce entropy.

Currently, a large amount of living material, known as phytoplankton (microorganisms such as cyanobacteria and diatoms), float on the surface of Earth's oceans. Besides these, there are also many viruses and free floating RNA and DNA fragments, pigments, lipids, and other organic compounds at the surface. This set of microorganisms and molecules absorb an important part of sunlight and convert this light into heat which is then transmitted to the surface water and air. If the ocean surface was devoid of organic material, only the infrared and the far ultraviolet light would be absorbed strongly by the surface water [25], with the rest of the solar spectrum being reflected and transmitted to space or to deeper ocean depths. The existence of this soup of microorganisms, organic molecules, and water at the surface thus allows not only the absorption of the infrared and far ultraviolet light, but also the visible and the near ultraviolet parts of the solar spectrum. The heat of photon dissipation at the surface then turns liquid water into vapor (evaporation) which can then participate in the water cycle, a second coupled dissipative process, further increasing the shift of the incident light towards the infrared, i.e. increasing global entropy production [12].

Evaporation produces clouds which are blown inland and cause rain on land. Minerals washed out of the rocks by the rain foment the growth of terrestrial plants that absorb sunlight better than bare sand and rock through organic pigments such as chlorophyll [24]. Some of the minerals washed out of rocks on land end up in rivers which eventually flow into the oceans where these nutrients stimulate greater phytoplankton growth. The absorption of sunlight through organic molecules at the ocean surface thus promotes the water cycle and the water cycle in turn stimulates the growth of life on both land and the ocean surface [12]. This phenomena of the autocatalytic coupling of different irreversible thermodynamic processes (photon absorption in phytoplankton and plants with the water cycle) is a form of more complex dissipative structuring which is part of the biosphere [11], and leads to greater global photon dissipation. The coupling of the dissipation of light by organic molecules on the Earth's surface with the water cycle accounts for more than $63 \%$ of all the entropy generated by the Sun-Earth interaction [26]. Most of the rest of the entropy production is due to light absorption on atmospheric molecules, which, in fact, also owe their existence primarily to life, e.g. water vapor, ozone, and water soluble organic dust.

Life, then, removes a restriction to entropy production on Earth by absorbing and dissipating light more efficiently and over a much wider spectral region, where, in fact, our Sun is most intense. Given that the dissipation of photons is the most relevant thermodynamic work performed by life today, it is very likely that this was also its function 3.85 billion years ago, a date for which there is evidence in the form of the ratio of carbon isotopes (life prefers to incorporate the lighter carbon isotope into its structures) that life originated [27].

Due to a lack of oxygen and ozone (before the invention of oxygenic photosynthesis), Earth's atmosphere at the time of the origin of life allowed the free passage of light within wavelengths between approximately 205 and 285 nanometers (nm), which is in the ultraviolet (long wavelength part of the UVC). At the same time, there was less near UV and visible light (between 320 and $700 \mathrm{~nm}$ ) due to the fact that the Sun's radius was smaller at the origin of life than today [28]. Photons in this long wavelength ( 205 to $285 \mathrm{~nm}$ ) part of the UVC region were ideally suited for dissipative structuring because they have enough energy to promote the breaking and remaking of covalent bonds of carbon based precursor molecules (like $\mathrm{HCN}$ and $\mathrm{CO}_{2}$ in water [23]), but not enough energy to successively ionize, and thereby destroy, these molecules.

It is conceivable, then, that life began as dissipatively structured pigments under UVC light to absorb and dissipate photons in this spectral region. The eventual dissipative structuring of the 
nucleobase adenine, and the related energy storage molecule adenosinetriphosphate (ATP), would allow dissipative structuring to occur under the higher intensity, but lower energy, visible wavelengths, by using, not one, but various visible photons together to transform molecules through the chemical potential stored in ATP. This would allow still more complex biosynthetic pathways to emerge based on visible photons, such as a primitive photosynthesis, leading to the dissipative structuring of still more pigments, now in the visible, and giving rise to new pathways and catalysts for photon dissipation, eventually leading to complex ecosystems, which corresponds to the organization of material in the form most efficient for photon dissipation found on Earth [24].

It must be emphasized that in our thermodynamic vision of life, biological evolution is not independent of other abiotic irreversible processes in the environment and that all coupled biotic-abiotic evolution is directed by the same thermodynamic imperative of increasing photon dissipation, or, in other words, entropy production.

\subsection{Evidence for the Theory}

RNA, DNA, and most of the fundamental molecules of life, efficiently absorb and dissipate UVC photons within a spectral region of about 205 to $285 \mathrm{~nm}$, just the wavelengths of the Archean atmospheric window (see Fig. 1). On exposure to UVC light, the nucleobases of RNA and DNA (adenine, guanine, cytosine and thymine or uracil) and also the other fundamental molecules of life, are electronically excited (collective excitation of electrons) and decay extremely rapidly ( $<2$ pico seconds, i.e. $2 \times 10^{-12} \mathrm{~s}$ ) through a process known as internal conversion, which converts the electronic excitation energy due to the absorbed photon rapidly into heat. The nucleobases do this through what is known as a conical intersection obtained by morphing into a very specific geometrical configuration once in their electronic excited state which allows the coupling of vibrational states superimposed on the electronic excited state to the vibrational states superimposed on the electronic ground state (see Fig. 2). This extremely fast dissipation of the electronic excitation energy through such a conical intersection makes RNA, DNA, and the other fundamental molecules of life (which also have them), extremely efficient at photon dissipation and stable against other photo-reactions that could further modify their structure. This is because reactions occur preferentially in the excited state and the lifetimes of these excited states for molecules with conical intersections are simply so short that not much else happens.

The extremely rapid de-excitation of the fundamental molecules also means, by the Heisenberg uncertainty principle $\Delta E \Delta t>\hbar$, that they also absorb over a wide wavelength region (i.e. $\Delta E$ is large since $\Delta t$ is small). As we will see, this has important consequences for the "thermodynamic selection" of these molecules over other possible configurations. 


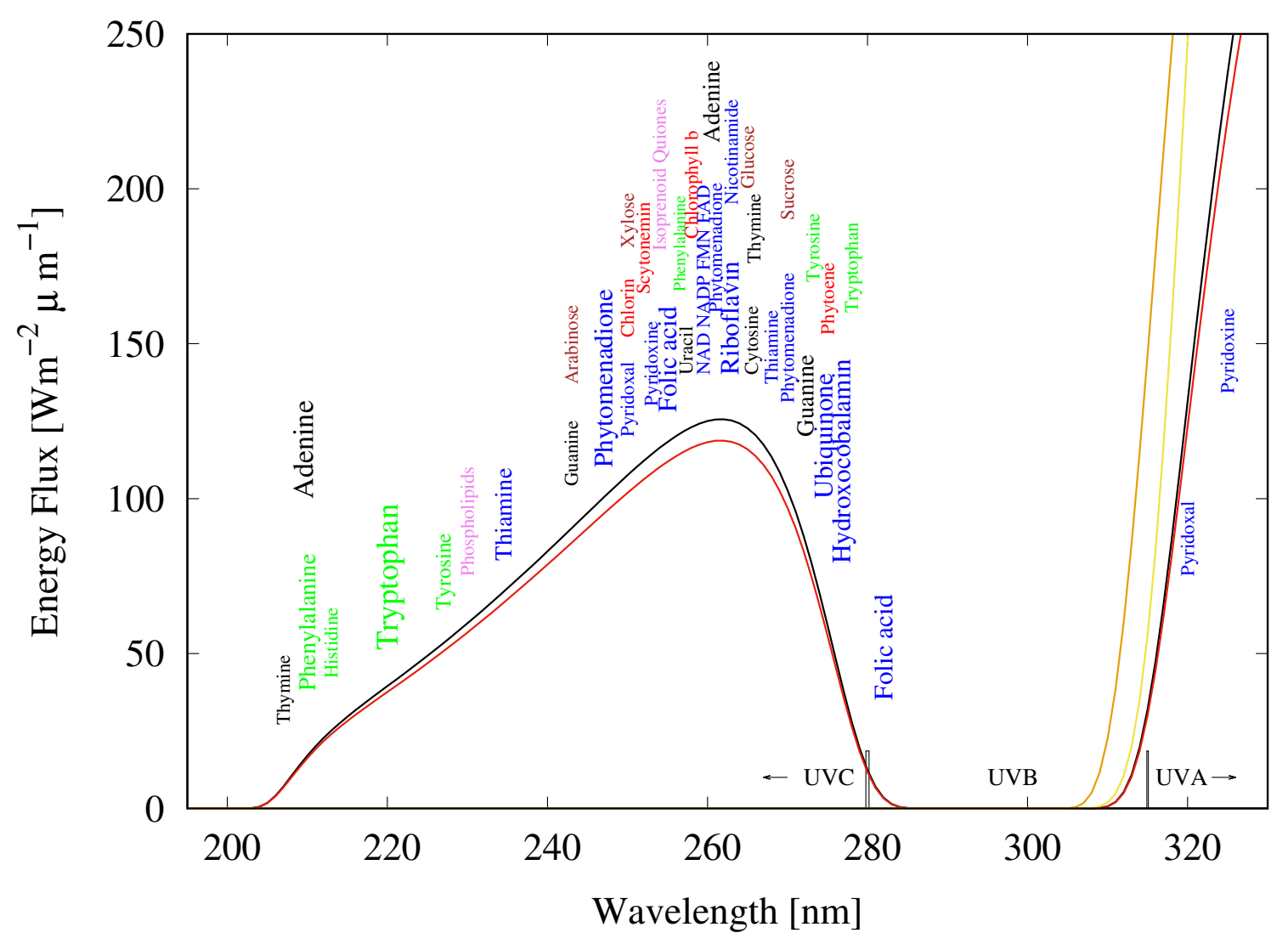

Figure 1. The spectrum of light available in the different UV regions at Earth's surface before the origin of life at approximately $3.85 \mathrm{Ga}$ and until at least $2.9 \mathrm{Ga}$ (curves black and red respectively) during the Archean. $\mathrm{CO}_{2}$ and probably some $\mathrm{H}_{2} \mathrm{~S}$ were responsible for absorption at wavelengths shorter than $\sim 205 \mathrm{~nm}$ and atmospheric aldehydes (comon photochemical products of $\mathrm{CO}_{2}$ and water) absorbed between about 285 and $310 \mathrm{~nm}$ [29], approximately corresponding to the UVB region. Around 2.2 Ga (yellow curve), UVC light at Earth's surface had been extinguished by oxygen and ozone resulting from organisms performing oxygenic photosynthesis. The green curve corresponds to the present surface spectrum. Energy fluxes are for the sun at the zenith. The names of the fundamental molecules of life, nucleic acids (black), amino acids (green), fatty acids (violet), sugars (brown), vitamins, co-enzymes and co-factors (blue), and pigments (red) are plotted at their wavelengths of maximal absorption (the font size of the letter roughly corresponds to the relative size of their molar extinction coefficient, i.e. how well they absorb). Note how closely these wavelengths of maximum absorption coincide with the Archean UV surface spectrum. Adapted from Michaelian and Simeonov [14]. 


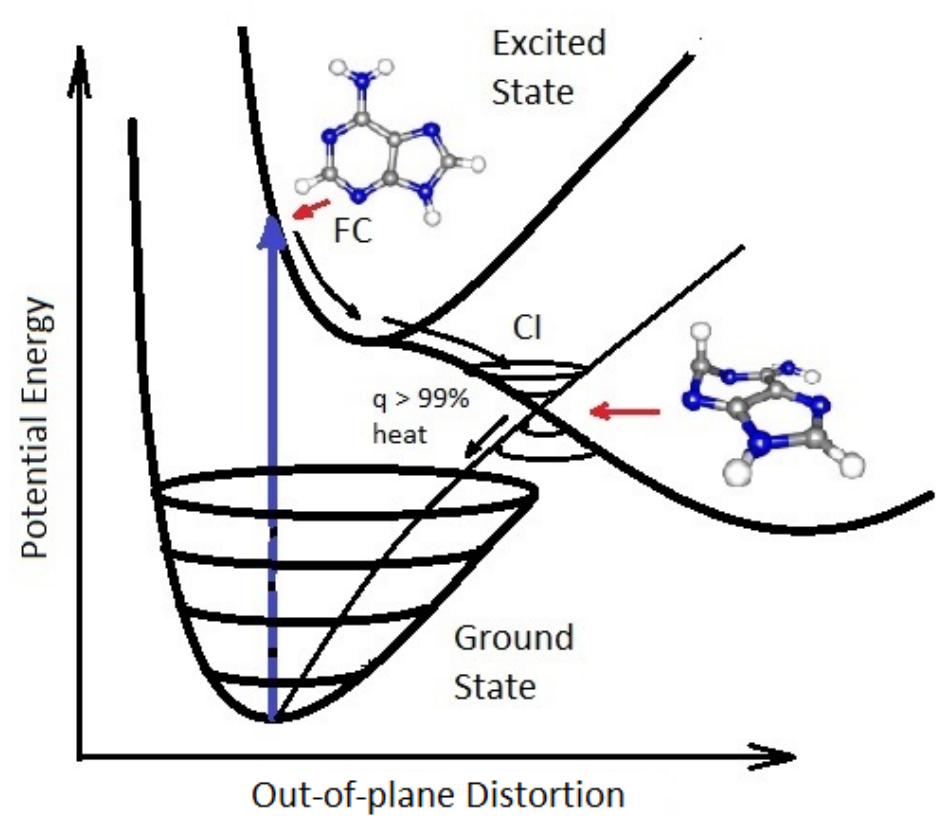

Figure 2. Conical Intersection (CI) for adenine (one of the nucelobases) showing the degeneracy of the electronic excited state with the electronic ground state after a UVC photon absorption event (blue arrow) which induces a nuclear coordinate deformation, known as pyrimidilization, from its original structure in the Franck-Condon (FC) region. In this deformed state, the vibrational states of the excited potential energy surface coincide in a region $(\mathrm{CI})$ with the vibrational states on the ground state. Conical intersections provide rapid (sub-picosecond) dissipation of the original electronic excitation energy into heat. The quantum efficiency $(\mathrm{q})$ for this dissipative route is very large, making adenine photochemically stable but, more importantly, very efficient at photon dissipation. Another common form of coordinate transformation associated with conical intersections are proton transfers within the molecule or with the solvent. Based on data from Andrew Orr-Ewing [30] Roberts et al. [31], Kleinermanns et al. [32] and Barbatti et al. [33]. Taken with permission from Michaelian [23]

The strong absorption of the fundamental molecules over the UVC spectral region arriving at Earht's surface during the Archean, and the fact that many of the fundamental molecules also have a conical intersection for the rapid dissipation of the absorbed energy into heat, is strong evidence that the fundamental molecule were originally UVC pigments and microscopic dissipative structures.

Furthermore, there exists empirical evidence suggesting selection for traits optimizing UV exposure for particular amino acids complexed with their RNA or DNA cognate codons or anticodons, particularly for those amino acids displaying the strongest stereochemical affinity to DNA and RNA. Such amino acids are, for example, aromatic amino acids, which could have acted as auxiliary antennas to DNA and RNA for greater UVC light dissipation, and amphiphilic (having one part hydrophilic and the other part hydrophobic) amino acids acting as anchors for DNA and RNA to the ocean surface where light intensity is maximal. This has led us to suggest [34] that UVC photon dissipation was the basis of the initial specificity in the amino acid - nucleic acid association during an early stereochemical era [35]. If this were the case, the information content of DNA and RNA would have been initially related to optimizing UVC photon dissipation. Today, the collective information content of the DNA and RNA of all living organisms is related to optimizing photon dissipation from the UVC, through the visible, and up to the red-edge.

Perhaps the most convincing evidence of all, however, is that many photochemical routes to the synthesis of nucleic acids, amino acids, fatty acids and sugars from simple, presumably common, precursor molecules (such as $\mathrm{H}_{2} \mathrm{O}, \mathrm{HCN}$, and $\mathrm{CO}_{2}$ ) have been identified at these wavelengths (205-285 
$\mathrm{nm}$ ) and the rate of photon dissipation within the Archean atmospheric window generally increases after each incremental step on route to synthesis [16,20], a behavior strongly suggestive of dissipative structuring (see section 2).

In the following, we present a brief overview of the microscopic dissipative structuring of one class of fundamental molecules of life, the nucleic acids. More details, and the dissipative structuring of the other classes, e.g. the fatty acids, is given elsewhere [20,23].

\subsection{The Dissipative Structuring of the Nucleic Acids}

In 1924 Alexander Oparin published a treaties on the origin of life [36] in which he suggested production of life's molecules from an atmosphere of reducing gases like hydrogen, ammonia, and methane, subjected to the heat and electric discharge from lightening. Similar ideas were also developed by John Haldane in 1929 who put forward the idea of a primordial organic soup [37].

In 1953 Stanley Miller proposed to his graduate adviser Harold Urey that they follow Oparin's recipes and designed and conducted experiments [38,39] of heating of, and electrical discharges on, a reducing atmosphere containing ammonia, methane, hydrogen, and water and confirmed the production of 5 amino acids; glycine, $\alpha$-alanine, $\beta$-alanine, aspartic acid and $\alpha$-aminobutyric acid. Recently, Jeffrey Bada (a student of Miller) performed a reanalysis with modern equipment of Miller's products (including some results obtained by using an atmosphere of $\mathrm{H}_{2} \mathrm{~S}$ and $\mathrm{CO}_{2}$ preserved for all these years in glass flasks). Bada showed, in fact, that not only 5, but many of the 22 amino acids used by life were produced in the Miller experiments [40].

More recently, however, the scientific community has come to the consensus that the atmosphere at the beginning of the Archean, when life arose, was probably not as reducing as proposed in the Miller-Urey experiments. It is now suggested instead that the early atmosphere was produced by oxidized gasses coming from the magma of volcanoes, as evidenced from the degree of oxidation of metals contained within sturdy zicron crystals of the era. Therefore, some other form of production of the fundamental molecules from a more neutral atmosphere was probably more likely, although induced lightening strikes on $\mathrm{H}_{2} \mathrm{~S}$ and $\mathrm{CO}_{2}$ within gas and ash clouds coming from volcanic eruptions remained a possibility for the production of the amino acids containing sulfur.

Experiments by Óro and Kimball [41] as well as those by Ferris and collaborators [42] were able to obtain not only simple amino acids, but also the RNA and DNA purine and pyrimidine nucleobases with only HCN in water at high temperature. In 1966 of Ferris and Orgel [43] using UV light were also able to obtain a copious production of the purine nucleobase adenine with HCN in warm water. The fact that many of these fundamental molecules have now also been found in meteorites, on the surface of comets, and even in the very cold interstellar gas and dust clouds, hints at the utility of UV light as a free energy source for their production under a myriad of different physical conditions. There remains, of course, an important question to resolve as to which chemical or photochemical routes were the actual routes that brought these first molecules to the surface of Earth.

An important clue to answering this question was given in the Introduction in that life is much more than a simple collection of fundamental molecules arranged in a particular pattern. Life manifests a vitality as seen in macroscopic dynamical processes such as; proliferation, mobility, metabolism, homeostasis, increases in complexity, and the ability to evolve and adapt to different environments. Unraveling this dynamical aspect of life by exploring the non-equilibrium thermodynamics of open systems should help answer the question of the actual routes to the fundamental molecules.

Lars Onsager (Nobel Prize in Chemistry 1968) and Ilya Prigogine (Nobel Prize in Chemistry 1977), starting from the original idea of Boltzmann, that the ordering of material in space and time, what we call "dynamical processes" or "the vitality of life", could only happen with a concomitant greater probability distribution of energy over microscopic degrees of freedom in another connected space-time region (the environment), showed that dissipative structuring often occurs to foment this process in non-linear systems held under an external force which keeps the system from reaching equilibrium. 
In fact, just as a non-linear equation has various possible solutions, non-linear relations between the forces and flows, implies that the system has available to it more than one particular structuring or state. Those states or structures (organization of material in space), or more correctly, those processes (organization of material in space and time) that disperse more efficiently the conserved quantities of nature over the microscopic degrees of freedom of the material of the environment can be shown to be the most stable [23], and thus we will most likely find open systems in the higher entropy producing states. This also means that natural fluctuations in the environment, or within the system itself, could cause the system to leave its initial state and evolve towards those more stable states (configurations) that produce greater dissipation of the free energy incident from the environment.

An example of one such system in which two stable states exist for the system under the same imposed external force is the Bénard cell. This system consists of a liquid sandwiched between a lower plate at high temperature and an upper plate at lower temperature, and under the force of gravity (see figure 3). At small temperature gradients, the system is in the linear regime and a static homogeneous state results with only conduction of heat through the liquid from the lower plate. As the temperature gradient is increased, the system arrives at the non-linear regime (between force temperature gradient, and flow - of heat) and convection cells become physically possible (the upward boyant force of hot water overcomes the opposing frictional force due to viscosity). In this regime, there exists two states for the system at the same value of the impressed external force (temperature gradient), one in which the hot liquid comes up through the center of the cells and the other in which it comes up around the edges of the cells. The two states have different entropy production or, in other words, a different rate of the dissipation into the environment of the incident free energy coming from the temperature difference between the two plates. The state in which the hot liquid comes up through the center of the cell is the one with the greatest heat flow and thus more resistant to material or temperature fluctuations, and this more stable state is, in fact, the state of greatest entropy production. This is, therefore, the state that we should expect to encounter in nature most frequently, and, in fact, experiment verifies this. 


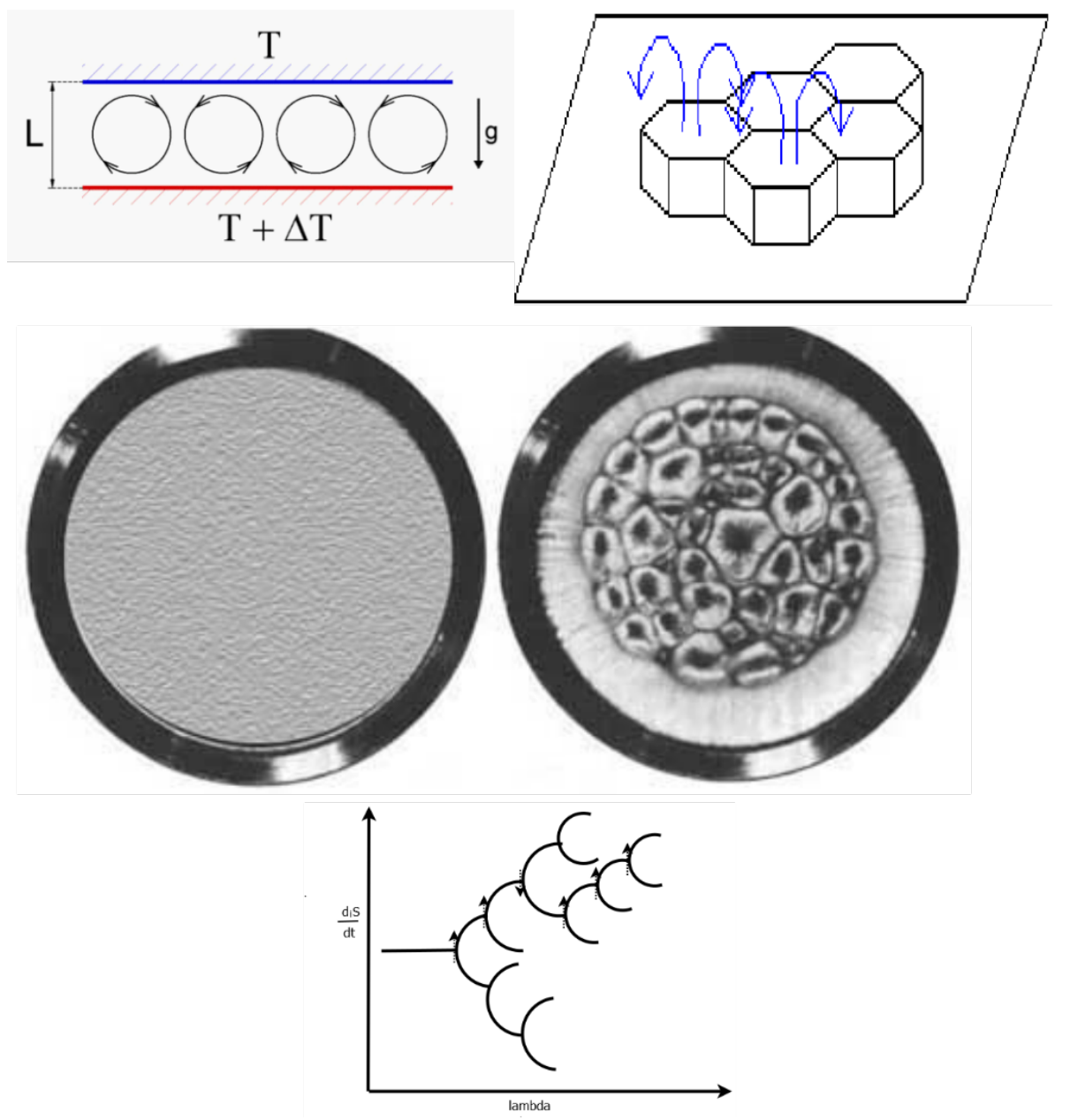

Figure 3. The Bénard cell. The system consists of a hot lower plate and a cold upper plate which sandwiches a liquid and the whole system is under the force of gravity. The onset of self-organization (structuring of material in space and time) occurs as a result of an external force (temperature gradient) imposed over the system, and a non-linear relation between the flow of heat and the temperature gradient. The left part of the figure 3 (a) describes the linear situation for the relation between the force (temperature gradient) and the flow (heat flow) when the temperature difference over the system is small, below some critical value $\Delta T_{C}$ and results in a homogeneous system (no spatial symmetry breaking) and there exists only conduction of heat. The right part of (a) describes the non-linear situation when the temperature difference between the two plates is greater than some critical value $\Delta T_{\mathcal{C}}$ for which convection cells spontaneously arise (Bénard cells) and spatial symmetry breaking occurs. There are two stationary states allowed for this system once the critical temperature gradient is reached; one with the hot liquid coming up through the center, and the other with the hot liquid coming up around the edges of the approximately hexagonal cells. The greater entropy producing $d_{i} S / d t$ state is the one with the hot liquid coming up through the center of the cells and this is the state of greater stability and thus the most probable given internal and external fluctuations of the material flow and temperature. This is shown in the bifurcation diagram (b) which describes the direction taken by the system in terms of entropy production as given by the arrows. The general trend is towards greater entropy production.

The same dynamics, we argue, is true for the case of microscopic dissipative structuring of the molecules of life in a system consisting of precursor molecules in water under a UVC photon flux, such as the spectrum given in figure 1 (see figure 4). Many different states, corresponding to different concentration profiles of the different possible intermediate and final product molecules, exist [23]. Those molecular concentrations profiles which are the most efficient at dissipating UVC photons, i.e. 
having greater absorption over a greater wavelength region in the UVC and greater emissivity in the infrared, i.e. characteristics most like a black-body [24]) are those states (concentration profiles) that are most resistant to fluctuations and thus these are the most probable, "statistically selected" concentration profiles in this non-equilibrium thermodynamic sense. The microscopic details of exactly how this occurs in the "self-organization" of the nucleobase adenine from HCN in water under UVC light, are given elsewhere $[16,23]$.

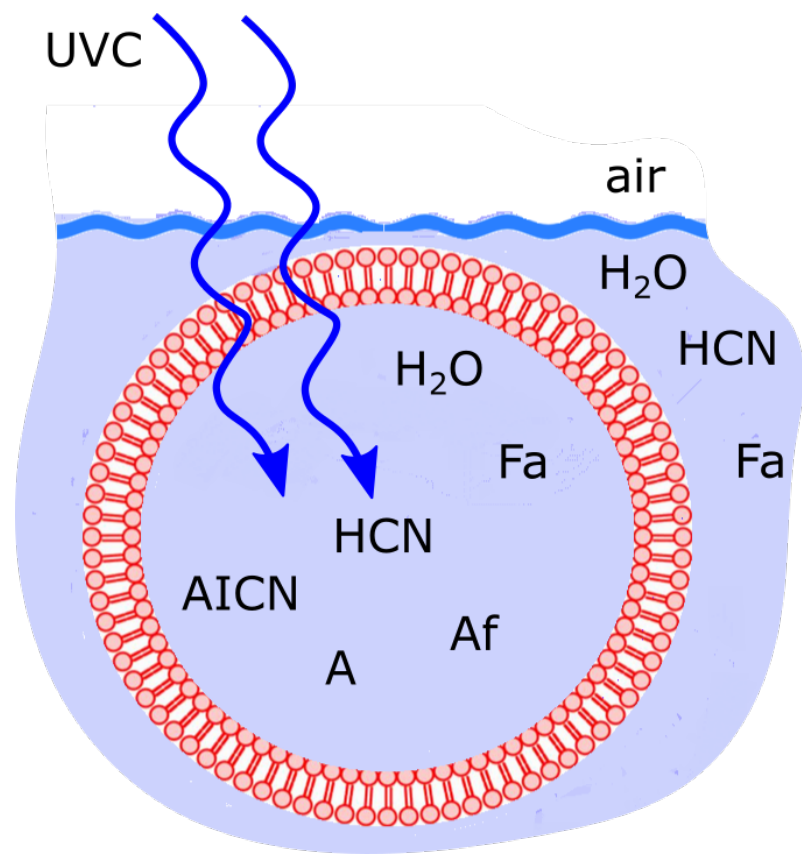

Figure 4. Fatty acid vesicle floating at the ocean surface microlayer, transparent to UVC light and permeable to the small molecules $\mathrm{H}_{2} \mathrm{O}, \mathrm{HCN}$ and formimidic acid (Fa) but impermeable to the photochemical reaction products (e.g., ammonium formate (Af), $\mathrm{AICN}$, adenine (A)) which are larger in size and have larger dipole moments. Concentration profiles of the intermediate and final product molecules which are most apt at dissipating the incident UVC photon flux are the most stable and therefore statistically selected in this non-equilibrium thermodynamic sense. Taken with permission from Michaelian [23].

Early life may thus be identified with a particular form of non-equilibrium structuring; microscopic dissipative structuring of carbon based molecules under UVC light leading to a particularly efficient photon dissipative concentration profile. The synthesized products, the fundamental molecules of life, are thus created as UVC pigments endowed with peaked conical intersections which gave them stability for long periods due to the reduced quantum efficiency for dexcitation through further photochemical reaction pathways. Unlike macroscopic dissipative structures such as hurricanes or convection cells, at normal Earth temperatures these microscopic dissipative structures remain intact even after the removal of the imposed light potential driving their synthesis due to strong covalent bonding between atoms.

\subsection{Proliferation and Selection}

In autocatalytic chemical reactions (those in which one of the reaction products acts as a catalyst for the reaction), and for systems kept out of equilibrium by a continuous supply of precursor reactant molecules to the system, the concentration of the product catalyst within the system can increase many orders of magnitude over the concentration expected for a system near equilibrium. Over 50 years ago, Ilya Prigogine [8] demonstrated, through classical irreversible thermodynamic (CIT) theory, that this result has its origin in the non-linear relationship between forces and corresponding flows (the forces are the affinities divided by the temperature and the flows are the reaction rates) and results in system 
evolution towards greater entropy production. That Nature favors these autocatalytic or cross-catalytic reactions can be imagined quite easily since a catalyst increases the rate of the reaction and thus if the reaction is autocatalytic the rate of catalyst production will increase accordingly, a positive feed back loop. This also increases the rate of dissipation of the chemical potential (entropy production), thereby reducing the amount of chemical potential available to competing reactions.

The situation is similar for the photochemical reactions in the dissipative structuring that we suggest led to the origin of life. If intermediate product molecules on route to the dissipative synthesis of the fundamental molecules act as catalysts for the photochemical or chemical reactions, then this would lead to their proliferation, as well as to that of their final product molecules. Those intermediate or final product molecules which are most rapid in dissipating the photon-induced excited state energy into heat will, by the Heisenberg uncertainty principle, absorb over a greater wavelength region and thereby reduce the available photochemical potential to other competing photochemical reactions. These photochemical reactions will therefore be the most probable (having largest quantum efficiency) and have greatest photon dissipation efficacy (entropy production). Efficacy for dissipation is therefore selected for, and this, along with proliferation, provides a mechanism for evolution which may be termed dissipative selection, or more generally, thermodynamic selection.

\subsection{Nucleic Acid Polymers}

We have explained how the fundamental molecules of life were dissipatively structured into UVC pigments from $\mathrm{HCN}, \mathrm{CO}_{2}$ and $\mathrm{H}_{2} \mathrm{~S}$ in water under UVC light. This "spontaneous" re-organization of covalently bound atoms in molecules under UVC light leading to dissipative UVC pigments is in analogy to the formation of a convection cell, or hurricane, under a temperature gradient. We also explained how these molecules proliferated over Earth's surface because of their auto-catalytic and cross-catalytic nature in fomenting photochemical reactions leading to the same molecules, and we have suggested how intermediate and final product molecules dissipating more photons than others will be more stable (selected) under the incident photon flux. But, why and how did the nucleobases polymerize into RNA and DNA nucleic acids, and how and why were these polymers faithfully replicated? If our postulate of life's origin being tied to dissipation is to be consistent, all processes, including the polymerization of the nucleotides into nucleic acid, and the replication of these, must also be explainable in terms of increasing photon dissipation.

It can be imagined that the nucleobases and other fundamental molecules formed within an out-of-equilibrium photochemical reaction system consisting of a fatty acid vesicle floating within the surface microlayer of a hot $\left(\sim 80^{\circ} \mathrm{C}\right.$ [44-46]) Archean ocean surface under the UV spectrum of Figure 1 (see Figure 4). Fatty acid vesicles form spontaneously on the ocean surface and we have demonstrated elsewhere how the long-chain fatty acids themselves can be spontaneously dissipatively structured from $\mathrm{CO}_{2}$ in water under UVC light [20]. The fatty acid vesicle is permeable at these high temperatures to small precursor molecules like $\mathrm{HCN}, \mathrm{CO}_{2}$ or $\mathrm{H}_{2} \mathrm{~S}$, but impermeable to the larger products like the nucleobase adenine. Without going into the chemical details, since they have been presented elsewhere $[15,20,23]$, we assume that the nucleobases formed nucleosides with ribose sugar and could have been activated (phosphorylated) in the presence of phosphates under UVC light, forming the nucleotides, for which there is experimental evidence $[47,48]$.

Because of pi-interactions between the aromatic rings of the nucleobases (a van der Waals interaction) and because of hydrophobic forces, there will be some natural stacking of the nucleotides, one on top of the other, in a water solvent, allowing phosphodiester bonds to form between a phosphate group and the $3^{\prime}$ and 5' carbon atoms of two adjacent sugars to link and form the sugar-phosphate backbone of DNA or RNA. Hydrolization of two phosphate groups, gives enough energy to overcome the barrier for formation of the phosphodiester bond.

Once the bases are polymerized, they are significantly more stable than when they are isolated [49], however, this alone cannot explain the polymerization since the Gibbs free energies are higher in 
the polymerized state. For consistency, we need an explanation from non-equilibrium thermodynamics related, again, to improvements in dissipation.

It is well known that some organic molecules can act as acceptor quencher molecules to the electronic excitation energy of donor molecules if the acceptor and donor molecules are in close proximity [50]. As mentioned above, the bases, as well as the polymers RNA and DNA, are superb quenchers, dissipating their electronic excitation energy within sub-picosecond times. They could, therefore, have acted as acceptors to quench the UVC induced excitation energy of other fundamental molecules of life having a large UVC absorption cross section but slower non-radiative decay times or large quantum yields for radiative decay such as fluorescence, both of which reduce the efficiency of dissipation. For example, the aromatic amino acid tryptophan absorbs strongly in the UVC but has a long non-radiative decay time of nanoseconds and a probability of getting trapped into an even longer lived triplet spin state of about $20 \%$ (leading to phosphorescence) compared to the probability for RNA and DNA doing the same of about $0.01 \%$ [51,52]. Intercalation of tryptophan with single strand DNA, involving the stacking of the tryptophyl ring with the base ring, is known from monomer studies to lead to the total quenching of both the tryptophan and the base fluorescence [53,54]. In double strand DNA, the quenching persists on intercalation and the double strand opens somewhat at the site of the tryptophan [55].

The donor-acceptor complex of tryptophan together with RNA or DNA would have thus constituted a greater dissipating system than the sum of its individual molecular components and would therefore have been thermodynamically selected through a dissipation-replication mechanism to be described in the following section. Certain linear segments of native RNA and DNA having a particular base sequence do indeed have chemical affinity to tryptophan and to other UVC dissipating molecules. In fact, we have shown that codons and anti-codons (contiguous triplets of base pairs, each coding for a particular amino acid) of RNA and DNA, have strong chemical affinity to their respective UVC antenna aromatic amino acids, as well as to other dissipation fomenting amino acids such as the amphiphilics (one part hydrophobic and the other part hydrophylic) which would anchor RNA and DNA to the ocean surface where the UVC light would be most intense [22,35].

Polymerization of the bases would thus have provided a scaffolding for the attachment of other fundamental UV-C dissipating molecules, and this, through the donor-acceptor mechanism, would have led to a greater dissipating complex than the sum of its parts and so be thermodynamically selected. Besides leading to greater dissipation, since the affinity of the donor chromophore molecule to the RNA or DNA acceptor is selective to only particular codons, then this dissipation-replication mechanism would also have led to information accumulation in RNA and DNA (information for optimizing dissipation) and to a thermodynamic advantage, in terms of photon dissipation, to evolving a faithful replication mechanism [22].

\subsection{Polymer Replication}

The nucelobases gained more stability once polymerized and served as scaffolding, and electronic excitation quenchers, for other fundamental molecules which acted as antennas, such as the aromatic amino acids, or anchors to the ocean surface, such as amphiphilic amino acids. A microscopic ecosystem of DNA-amino acid molecular complexes thus began to establish itself on Earth's ocean surface to efficiently dissipate the UVC photons. How would only those complexes most efficient at dissipation be selected for replication and dispersal over the entire of Earth's ocean?

Recall that DNA forms double helix chains that remain bound thanks to the hydrogen bonds that are formed between the nitrogenous bases and the stacking interaction between adjacent bases. When these polymers are in water and the temperature is increased, the double strands of DNA separate into single strands at a particular temperature known as its denaturing temperature. In general, the denaturing temperature of each DNA and RNA double strand depends on the amount of hydrogen bonds that it contains, and on the stacking interaction, and therefore, on the number of nitrogenous bases it has and the proportion of G-C base pairs (with 3 hydrogen bonds) compared to the number 
of A-T bonds (with 2 hydrogen bonds), and also on the $\mathrm{pH}$ and the amount of salt in the solvent. In general, for ocean-like environments, the denaturing temperature of intermediate length strands of DNA is higher than about $75{ }^{\circ} \mathrm{C}$ and about $40{ }^{\circ} \mathrm{C}$ for double strand RNA.

As was previously mentioned, Earth's average surface temperature at the time of the origin of life was about $80^{\circ} \mathrm{C}$, interestingly close to the denaturing temperature of intermediate length segments of DNA. With this fact in mind, it is now possible to imagine a physical mechanism, based on photon dissipation, for RNA and DNA replication once the temperature of the seas dropped below their denaturing temperatures. We call this mechanism "Ultraviolet and Temperature Assisted Replication" (UVTAR) and consists in the following processes;

1. During the day, the absorption of UV light by RNA and DNA and some visible light by organic matter floating on the surface of the sea, in addition to the strong absorption of infrared light by the water, caused the sea surface temperature to increase above the denaturing temperature of the RNA or DNA, and thus a double strand separated into two single strands. Currently, the diurnal temperature variation of the first 100 microns of the sea surface can be up to $5{ }^{\circ} \mathrm{C}$, with maximal temperature during mid-afternoon. When RNA and DNA denature, there is about a $35 \%$ increase in the absorption and dissipation of the photons, known as the hyperchromic effect. Once denaturing starts at one end of the double strand, it would, therefore, tend to be autocatalytic.

2. At night, as ocean surface temperatures drop below the denaturing temperature of nucleic acid, the single strands would act as templates for the aggregation of complementary UVC activated fragments, permitting extension and leading to the formation of a new generation of double strands. As has been shown in the laboratory [48], this could have be catalyzed by magnesium ions $\left(\mathrm{Mg}^{+2}\right)$ instead of the enzyme polymerase that currently facilitates extension inside the contemporary cell.

This process is depicted in figure 5 and is similar to a technique commonly used in the laboratory to amplify (multiply) DNA known as the Polymerase Chain Reaction, or, simply PCR. Our process, however, uses the absorption of UVC light rather than heat to denature. This mechanism of denaturing, UVTAR, is not hypothetical, we have shown experimentally that it occurs for DNA and we have measured its temperature dependence [13]. 


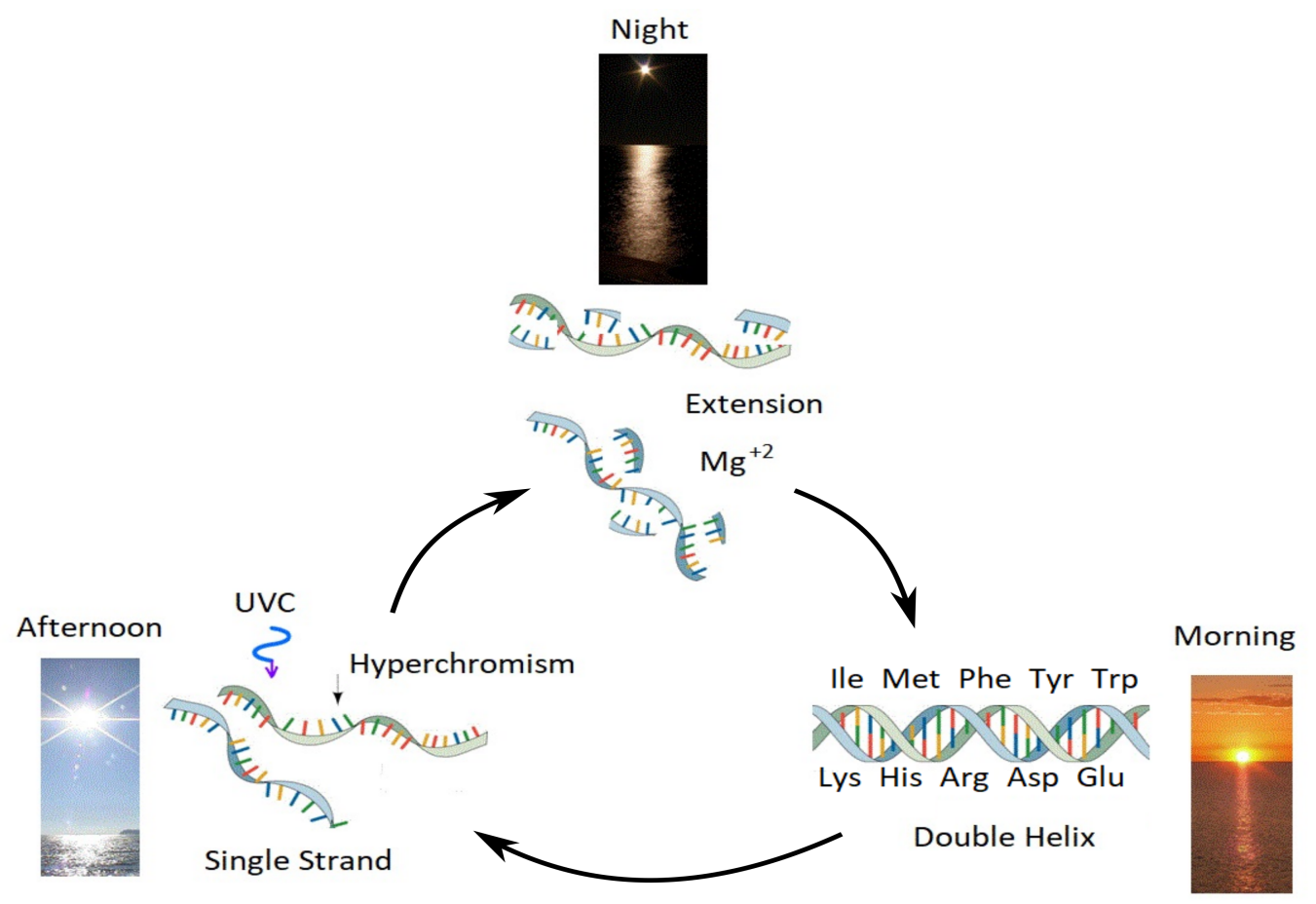

Figure 5. Ultraviolet and Temperature Assisted Reproduction (UVTAR) of RNA and DNA. A mechanism proposed for the enzyme-less reproduction of RNA and DNA assisted by the absorption and dissipation of the prevailing UVC light flux and the high temperatures of the ocean surface during the late Hadean or early Archean, including a day/night diurnal warming and cooling cycle of the water surface. Due to the absorption of solar infrared light during the day, most denaturing would occur in the afternoon when ocean surface temperatures were highest. This would lead to selection of a particular chirality (see text) [18]. Extension occurs overnight with the aid of $\mathrm{Mg}^{+2}$ ions, UVC activated nucleotides, and colder surface temperatures. "Hyperchromism" refers to an increase $(\sim 35 \%)$ in the absorption of photons at UVC wavelengths $(\sim 260 \mathrm{~nm})$ once RNA or DNA are denatured into single strands. Oligos which had chemical affinity to the 10 amino acids listed in the figure (all of which have photon absorption and dissipation fomenting characteristics [22]), would have had a greater chance of denaturing during daylight hours as the ocean surface temperature cooled throughout the Archean, and could therefore be replicated overnight. This selection based on greater photon dissipation we have termed "thermodynamic selection" $[9,10,15,23]$. The important aspect of this auto-catalytic mechanism is that replication is tied to photon dissipation, providing a thermodynamic imperative for proliferation. Taken with permission from [22].

\subsection{Evolution}

The thermodynamic dissipatiion theory for the origin of life suggests that natural selection is really thermodynamic selection that favors coupled biotic-abiotic systems of greater photon dissipation (global entropy production). The physical-chemical attraction between aromatic and amphiphilic amino acids listed in figure 5 and their cognate DNA, or RNA, codons (a stereochemical era), is an example of antenna-quencher association which foments replication of those complexes with efficient dissipation of the Archean UVC light.

It is therefore probable that the first information encoded in the RNA and DNA was that of the codons or anti-codons corresponding to these amino acids which promote dissipation. A greater photon dissipation rate would lead to a greater denaturing rate for the DNA or RNA strands that coded for these amino acids and thus to their greater replication rate, especially as the seas cooled throughout the Archean. Such thermodynamic selection at the molecular complex level then leads to the evolution of ever greater dissipative systems. 
Dissipative structuring under light, as the fundamental creative force in biology, appears to have been ongoing, from the initial dissipation at the UVC wavelengths of the Archean by the fundamental molecules of life, to the dissipation of wavelengths up to the red edge $(\sim 700 \mathrm{~nm})$ by the organic pigments of today $[9,10,14]$. Beyond the red edge, starting at about $1200 \mathrm{~nm}$, water in the ocean surface microlayer absorbs strongly and dissipates photons into heat efficiently. There is, therefore, still a wavelength region between 700 and $1200 \mathrm{~nm}$ which remains to be conquered by future evolution of pigments. The simultaneous coupling of biotic with abiotic irreversible processes, such as the water cycle and ocean and air currents, culminating in an efficient global dissipating system known as the biosphere, increases further the efficacy of solar photon dissipation into the far infrared much beyond $1200 \mathrm{~nm}[9,11,12]$.

Empirical evidence for selection in nature towards states of increased dissipation exists on vastly different size and time scales. For example, the increase in photon absorption and dissipation efficacy of a plant leaf over its life-cycle [56], the proliferation of photon absorbing pigments over the entire surface of Earth, the correlation between ecosystem succession and increased dissipation [57,58], and the general increase of biosphere efficacy in photon dissipation over evolutionary history, including, for example, the plant-induced increases in the water cycle [11,59] and animal dispersal of nutrients required for pigment synthesis [15]. There is also evidence for this at the microscopic scale, for example in the increased rates of energy dissipation per unit biomass of the living cell over its evolutionary history [60].

\subsection{The Homochirality of Life}

Perhaps one of the most difficult facts to explain concerning the origin of life is the homochirality of the fundamental molecules. Molecules having an $\alpha$-carbon atom come in two versions identical in all respects except for their chiral symmetry which affects the strength of their absorption of circularly polarized light. For example, all DNA and RNA from living organisms absorb preferentially right-hand circular polarized light, while the amino acids absorb preferentially left-hand circular polarized light. We say then that the DNA and RNA of living beings are right-handed and the amino acids are left-handed. Both versions of chirality are produced equally in abiotic experiments, but only one chirality is found in Nature.

Theories developed to explain homochirality have relied on either an initial preponderance of one chirality, e.g. through a selective destruction of the other chirality, or the chance formation of the original fundamental molecules on chiral inorganic substrates, or some kind of a asymmetric mechanism for the amplification of an initially very small chirality asymmetry. However, no theory has, so far, convincingly explained the homochirality of living systems.

Our theory offers a simple explanation of homochirality through the UVTAR mechanism for denaturing and reproduction of the nucleic acids (see figure 5) based on the fact that there is an important component of circularly polarized light at the ocean surface which is of one circular polarization in the morning and of the opposite in the afternoon. The ocean surface is, in fact, the region of greatest circular polarization of light on Earth [18]. The UVTAR mechanism for denaturing explained in section 3.5 is strongly temperature dependent, being more effective at higher temperatures [13]. In the afternoon, the Archean sea surface would have been about $3-5{ }^{\circ} \mathrm{C}$ warmer and thus greater denaturing of RNA or DNA would occur in the afternoon compared to the morning, therefore leading to the preferential denaturing and subsequent replication of one chirality. A few thousand years of such an asymmetric replication process would be sufficient to arrive at effectively $100 \%$ homochirality for the nucleic acids [18].

The stereochemical affinity of amino acids for nucleic acid is greater for opposite chiralities, for example, left-handed amino acids have greater affinity to right-handed RNA or DNA, and vice-versa. This could be sufficient to explain the left-handed chirality of the amino acids. 


\section{Comparison of Our Theory with other Proposals for the Origin of Life}

Metabolism is, of course, important for the maintenance and survival of every living organism. However, the amount of free energy dissipated in metabolism represents only a tiny fraction of all the free energy dissipated by life. The vast majority of the free energy that arrives in the photons from the Sun is simply dissipated into heat by organic pigments in water, without any utilization of this free energy by the organism. However, this apparently useless dissipation, as explained in this paper, is precisely the true essence of life, the thermodynamic reason for its existence. Traditional origin of life theories, recognize the need for a free energy source to maintain organism metabolism, perhaps obtained through chemical potentials or thermal gradients (such as at hydrothermal vents), but completely ignore the large amount of dissipation that life performs not related to metabolism. Such theories, therefore, lack an appreciation of the essence of life, which can only be understood within the framework of non-equilibrium thermodynamics.

During the Archean, UVC photons would have provided orders of magnitude more free energy for dissipation, or for precursor molecule carbon covalent bond transformation, than would have the chemical potentials used in thermal reactions. Sources of free energy available in chemical or thermal gradients would have been very limited in size and extent. Thermodynamically speaking, the dissipation of chemical or thermal gradients could, therefore, not compete with the dissipation of solar photon potential. Furthermore, a theory based on chemical or thermal gradients would have the very difficult problem of explaining the rapid occurrence of the process of photosynthesis very soon after the emergence of life with its unmistakable hallmark of photon dissipation (today, less than $1 \%$ of the light absorbed on phytoplankton or plants goes into the fixation of carbon, the rest is simply dissipated into heat by the photosynthetic and other systems).

The theory proposed here, like the theory of the origin of life at hydrothermal vents, supports a thermophilic (high temperature) origin of life, precisely what has been found by a careful comparative analysis of the genomes of primitive organisms [61], and also from an analysis of oxygen isotopes found in surface sediments of the era. Any irrefutable evidence indicating that the Earth surface was cold at the origin of life would probably make our proposed theory unfeasible.

The theory presented here views the origin of life as a thermodynamic imperative, the "self-organization" of a new irreversible process of organic pigment formation for the dissipation of photons and the coupling of the resulting heat of dissipation to other abiotic irreversible processes, in particular the water cycle. This irreversible process, which we call "life", appeared once the environmental conditions became appropriate about 3.85 billion years ago (e.g. surface UVC light and high temperatures) under the thermodynamic imperative of increasing the Earth's entropy production in its interaction with its solar environment. Such conditions have never again occurred on Earth's surface and this fact could explain the apparent emergence of life at only a single instant of time in Earth's history. Other theories would have to allow for the possibility that life could have arisen at numerous times throughout Earth's history, evidence for which is lacking.

\section{Conclusions}

The origin of life is one of the most enigmatic questions that has occupied the human imagination for millennia. Although it has been more than 150 years since Darwin, and later Oparin 100 years ago, suggested a physico-chemical origin of life, and more than 63 years since Miller and Urey's first experiments in 1959 that provided firm elements in favor of such a possibility, there is still no theory universally accepted by the scientific community. Life's complexity and the lack of understanding on how to reconcile biotic processes with abiotic physical processes has been an obstacle to progress. Out-of-equilibrium thermodynamics, however, offers a robust framework for the unification of biology and physics, while explaining the dynamics, or vitality, and complexity that arises from the non-linearity and the resulting bifurcations of dynamic solutions in systems kept away from the linear branch of thermodynamics by the imposed solar photon potential. 
The perspective taken here is that the origin of life was not a scenario of organic material organization driven by natural selection leading to "better adapted" organisms, or to greater chemically stability (e.g. UV resistant organisms), but rather a scenario of the dissipative structuring of material under the imposed UVC solar photon potential leading to an organization of material in space and time (biosynthetic pathways) providing more efficient routes to the dissipation of the externally imposed photon potential. The dissipative synthesis of an ever larger array of photochemical catalysts and cofactors, would mean that ever more complex biosynthetic pathways would emerge through thermodynamic selection on dissipation to promote the synthesis of novel pigments for dissipating not only the fundamental UVC and other UV regions, but eventually the entire short wavelength region of the solar photon spectrum $[14,17,23]$, eventually reaching the red-edge $(\sim 700 \mathrm{~nm})$ which is the approximate limit of biological photon dissipation on Earth today. According to our theory, thermodynamic evolution through dissipative selection is slowly pushing this red-edge ever further towards the infrared.

There is much empirical evidence for the existence of selection in nature towards states of increased dissipation on vastly different size and time scales. For example, the increase in photon absorption and dissipation efficacy of a plant leaf over its life-cycle [56], the proliferation of photon dissipating life over the entire Earth surface, the correlation between ecosystem succession and increased dissipation $[57,58]$, and the general increase of biosphere efficacy in photon dissipation (including, for example, the plant-induced increases in the water cycle $[11,59]$ and animal dispersal of nutrients required for pigment synthesis) over evolutionary history. There is also evidence for this at the microscopic scale, for example in the increased rates of chemical potential dissipation per unit biomass of the living cell over its evolutionary history [60].

Any planet around any star giving off light in the long wavelength UVC region, but with protection from short wavelength UVC light, should therefore have its own concentration profile of dissipatively structured carbon based fundamental molecules who's characteristics would depend on the exact nature of the local physical and UV environment and the precursor and solvent molecules available. We have considered the synthesis of such a basis set of dissipative molecules to be the first step of incipient life [15] and, therefore, under this criterion, incipient life has already been discovered on the other planets of our solar system, for example, in the sulfur containing UV pigments found in the clouds of Venus [62], the UV absorbing thiophenes [63] and red chlorophyll-like pigments [64] found on the surface of Mars, the UVC and UVB absorbing poly-aromatic hydrocarbons (PAHs) found in the atmosphere and on the surface of Titan [65] and in interstellar space [14]. The observation that thiophenes and PAHs found on Mars and in space are of generally large size can also be understood from our thermodynamic perspective since, without the possibility of vibrational dissipation through hydrogen bonding to solvent molecules, these molecules would have "grown" to large sizes through dissipative selection in order to support many low frequency vibrational modes which would increase the dissipation of the absorbed photon energy.

Dissipative structuring, dissipative proliferation, and dissipative selection, are the necessary and sufficient elements for a non-equilibrium thermodynamic framework from within which the origin and evolution of life can be explained in physical and chemical terms. Many details concerning the Thermodynamic Dissipation Theory for the Origin and Evolution of Life have been left out of this presentation simply because of lack of space, but the interested reader is encouraged to explore the following references in which the most important details are more thoroughly considered; $[9,10,14-16,21,23]$.

Funding: This research was funded by DGAPA-UNAM grant number IN104920.

\section{Acknowledgments:}

Conflicts of Interest: The author declares no conflict of interest.

\section{Abbreviations}


The following abbreviations are used in this manuscript:

A adenine

Af ammonium formate

AICN 4-aminoimidazole-5-carbonitrile

Arg amino acid arginine

Asp amino acid aspartic acid

CI conical intersection

CIT Classical Irreversible Thermodynamics

DAMN diaminomaleonitrile

DAFN diaminofumaronitrile

Fa formimidic acid

Glu amino acid glutamic acid

HCN hyrdorgen cyanide

His amino acid histidine

Ile amino acid isoleucine

Lys amino acid lysine

Met amino acid methionine

PAHs Polyaromatic Hydrocarbons

PCR Polymerase Chain Reaction

Phe amino acid phenylalanine

Tyr amino acid tyrosine

Trp amino acid tryptophan

UVA light in the region 360-400 nm

UVB light in the region $285-360 \mathrm{~nm}$ (only the region 310-360 $\mathrm{nm}$ is relevant here)

UVC light in the region 100-285 $\mathrm{nm}$ (only the region $210-285 \mathrm{~nm}$ is relevant here)

UVTAR Ultraviolet and Temperature Assisted Replication

\section{References}

1. Kauffman, S.A. Cellular Homeostasis, Epigenesis and Replication in Randomly Aggregated Macromolecular Systems. Journal of Cybernetics 1971, 1, 71-96, [https: / / doi.org/10.1080/01969727108545830]. doi:10.1080/01969727108545830.

2. Gilbert, W. The RNA world. Nature 1986, 319, 618.

3. Martin, W.; Baross, J.; Kelley, D.; et al.. Hydrothermal vents and the origin of life. Nature Reviews Microbiol. 2008, 6, 805-814.

4. Bernal, J.D. The physical basis of life. The Proceedings of the Physical Society Section A 1949, 62, 537-558.

5. Wigner, E.P. Physics and the explanation of life. Found Phys 1970, 1, 35-45. doi:10.1007/BF00708653.

6. Boltzmann, L. Ludwig Boltzmann: Theoretical physics and philosophical problems: Selected writings; Springer, 1974.

7. Boltzmann, L. Über die Methoden der theoretischen Physik; Vieweg+Teubner Verlag, 1979.

8. Prigogine, I. Introduction to Thermodynamics Of Irreversible Processes, third ed.; John Wiley \& Sons, 1967.

9. Michaelian, K. Thermodynamic origin of life. ArXiv 2009, [arXiv:physics.gen-ph/0907.0042]. doi:10.5194/esd-2-37-2011.

10. Michaelian, K. Thermodynamic dissipation theory for the origin of life. Earth Syst. Dynam. 2011, 224, 37-51, [https:/ / esd.copernicus.org/articles/2/37/2011/esd-2-37-2011.html].

11. Michaelian, K., The Biosphere; INTECH, 2012; chapter The biosphere: A thermodynamic imperative.

12. Michaelian, K. Biological catalysis of the hydrological cycle: lifes thermodynamic function. Hydrol. Earth Syst. Sci. 2012, 16, 2629-2645, [www.hydrol-earth-syst-sci.net/16/2629/2012/]. doi:10.5194/hess-16-2629-2012.

13. Michaelian, K.; Santillán Padilla, N. DNA Denaturing through Photon Dissipation: A Possible Route to Archean Non-enzymatic Replication. bioRxiv 2014, [https://www.biorxiv.org/content/early/2014/11/24/009126.full.pdf]. doi:10.1101/009126. 
14. Michaelian, K.; Simeonov, A. Fundamental molecules of life are pigments which arose and co-evolved as a response to the thermodynamic imperative of dissipating the prevailing solar spectrum. Biogeosciences 2015, 12, 4913-4937, [https:/ /bg.copernicus.org/articles/12/4913/2015/].

15. Michaelian, K. Thermodynamic Dissipation Theory of the Origina and Evolution of Life: Salient characteristics of RNA and DNA and other fundamental molecules suggest an origin of life driven by UV-C light; Self-published. Printed by CreateSpace. Mexico City. ISBN:9781541317482., 2016.

16. Michaelian, K. Microscopic Dissipative Structuring and Proliferation at the Origin of Life. Heliyon 2017, 3, e00424, [https://www.ncbi.nlm.nih.gov/pmc/articles/PMC5647473/]. doi:10.1016/j.heliyon.2017.e00424.

17. Michaelian, K.; Simeonov, A. Thermodynamic explanation of the cosmic ubiquity of organic pigments. Astrobiol. Outreach 2017, 5, 156, [https://www.longdom.org/open-access/thermodynamic-explanation-for-the-cosmic-ubiquity-of-organicpigments-2332-251

18. Michaelian, K. Homochirality through Photon-Induced Denaturing of RNA/DNA at the Origin of Life. Life 2018, 8, [http:/ / www.mdpi.com/2075-1729/8/2/21]. doi:10.3390/life8020021.

19. Michaelian, K. Photochemical Dissipative Structuring of the Fundamental Molecules of Life. Proceedings, 5th International Electronic Conference on Entropy and Its Applications; Session: Biological Systems 2019.

20. Michaelian, K.; Rodriguez, O. Prebiotic fatty acid vesicles through photochemical dissipative structuring. Revista Cubana de Química 2019, 31, 354-370.

21. Michaelian, K.; Santillan, N. UVC photon-induced denaturing of DNA: A possible dissipative route to Archean enzyme-less replication. Heliyon 2019, 5, e01902, [https:/ / www.heliyon.com/article/e01902].

22. Mejía Morales, J.; Michaelian, K. Photon Dissipation as the Origin of Information Encoding in RNA and DNA. Entropy 2020, 22, [https:/ / www.mdpi.com/1099-4300/22/9/940]. doi:10.3390/e22090940.

23. Michaelian, K. The Dissipative Photochemical Origin of Life: UVC Abiogenesis of Adenine. Entropy 2021, 23, [https:/ /www.mdpi.com/1099-4300/23/2/217]. doi:10.3390/e23020217.

24. Michaelian, K.; Mateo, R.E.C. A Photon Force and Flow for Dissipative Structuring: Application to Pigments, Plants and Ecosystems. Entropy 2022, 24, 76. doi:10.3390/e24010076.

25. Chaplin, M. Water Structure and Science 2016. [https://water.lsbu.ac.uk/water].

26. Kleidon, A. Maximum entropy production and general trends in biospheric evolution. Paleontological Journal 2009, 43, 980-985.

27. Schidlowski, M. A 3,800-million-year isotopic record of life from carbon in sedimentary rocks. Nature 1988, 333, 313-318.

28. Karam, P.A. Inconstant Sun: how solar evolution has affected cosmic and ultraviolet radiation exposure over the histroy of life on Earth. Health Phys. 2003, 84, 322-333.

29. Sagan, C. Ultraviolet Selection Pressure on the Earliest Organisms. J. Theor. Biol. 1973, 39, 195-200.

30. Orr-Ewing, A. Reaction Dynamics -Relaxation Pathways. Lecture Notes 2014, pp. 1-36.

31. Roberts, G.M.; Marroux, H.J.B.; Grubb, M.P.; Ashfold, M.N.R.; Orr-Ewing, A.J. On the Participation of Photoinduced N-H Bond Fission in Aqueous Adenine at 266 and $220 \mathrm{~nm}$ : A Combined Ultrafast Transient Electronic and Vibrational Absorption Spectroscopy Study. The Journal of Physical Chemistry A 2014, 118, 11211-11225, [https:/ / doi.org/10.1021/jp508501w]. PMID: 25296392, doi:10.1021/jp508501w.

32. Kleinermanns, K.; Nachtigallová, D.; de Vries, M.S. Excited state dynamics of DNA bases. International Reviews in Physical Chemistry 2013, 32, 308-342, [https://doi.org/10.1080/0144235X.2012.760884]. doi:10.1080/0144235X.2012.760884.

33. Barbatti, M.; Aquino, A.; Szymczak, J.; Nachtigallová, D.; Hobza, P.; Lischka, H. Relaxation mechanisms of UV-photoexcited DNA and RNA nucleobases. Proc Natl Acad Sci U S A 2010, 107, 21453-21458. doi:doi:10.1073/pnas.1014982107.

34. Mejía, J.; Michaelian, K. Information Encoding in Nucleic Acids through a Dissipation-Replication Relation. ArXiv 2018, [arXiv:physics.bio-ph/1804.05939]. doi:10.3390/e22090940.

35. Yarus, M.; Widmann, J.; Knight, R. RNA-Amino Acid Binding: A Stereochemecal Era for the Genetic Code. J Mol Evol 2009, 69, 406-429. doi:10.1007/s00239-009-9270-1.

36. Oparin, A.I., Proiskhozhdenie zhizny (The origin of life). In The origin of life; Bernal, J.D., Ed.; Weidenfeld and Nicholson, 1924.

37. S., H.J.B. Origin of life. Rationalist Annual 1929, 148, 3-10. 
38. Miller, S.L. A Production of Amino Acids Under Possible Primitive Earth Conditions. Science 1953, 117, 528-529, [https://science.sciencemag.org/content/117/3046/528.full.pdf]. doi:10.1126/science.117.3046.528.

39. Miller, S.L.; Urey, H.C. Origin of Organic Compounds on the Primitive Earth and in Meteorites. Science 1959, 130, 245.

40. Bada, J.L. New insights into prebiotic chemistry from Stanley Miller's spark discharge experiments. Chem. Soc. Rev. 2013, 42, 2186-2196. doi:10.1039/C3CS35433D.

41. Oró, J.; Kimball, A. Synthesis of purines under possible primitive earth conditions: II. Purine intermediates from hydrogen cyanide. Archives of Biochemistry and Biophysics 1962, 96, 293 - 313. doi:https:/ / doi.org/10.1016/0003-9861(62)90412-5.

42. Ferris, J.; Joshi, P.; Edelson, E.; Lawless, J. HCN: a plausible source of purines, pyrimidines and amino acids on the primitive Earth. Journal of molecular evolution 1978, 11, 293-311.

43. Ferris, J.P.; Orgel, L.E. An Unusual Photochemical Rearrangement in the Synthesis of Adenine from Hydrogen Cyanide. J. Am. Chem. Soc. 1966, 88, 1074-1074.

44. Karhu, J.; Epstein, S. The Implication of the Oxygen isotope Records in Coexisting Cherts and Phosphates. Geochim. Cosmochim. Acta 1986, 50, 1745-1756.

45. Knauth, L.P., Lecture Notes in Earth Sciences \#43; Springer-Verlag, Berlin, 1992; chapter Isotopic Signatures and Sedimentary Records, pp. 123-152.

46. Knauth, L.P.; Lowe, D.R. High Archean climatic temperature inferred from oxygen isotope geochemistry of cherts in the 3.5 Ga Swaziland group, South Africa. Geol. Soc. Am. Bull. 2003, 115, 566-580.

47. Ponnamperuma, C.; Sagan, C.; Mariner, R. Synthesis of adenosine triphosphate under possible primitive Earth conditions. Nature 1963, 199, 222-226.

48. Szostak, J.W. The eightfold path to non-enzymatic RNA replication. J. Sys. Chem. 2012, 3, 2.

49. Saladino, R.; Crestini, C.; Ciciriello, F.; Di Mauro, E.; Costanzo, G. Origin of informational polymers: Differential stability of phosphoester bonds in ribomonomers and ribooligomers. J. Biol. Chem. 2006, 281, 5790-5796.

50. Vekshin, N. Photonics of Biopolymers, first ed.; KomKniga, Moscow, 2005.

51. Bensasson, R.V.; Land, E.J.; Truscott, T.G. Flash Photolysis and Pulse Radiolysis: Contributions to the Chemistry of Biology and Medicine; Pergamon Press, 1983.

52. Cadet, J.; Vigni, P. Bioorganic Photochemistry: Photochemistry and the Nucleic Acids; John Wiley \& Sons, 1990; pp. 1-273.

53. Montenay-Garestier, T.; Helene, C. Molecular Interactions between Tryptophan and Nucleic Acid Components in Frozen Aqueous Solutions. Nature 1968, 217, 844-845.

54. Montenay-Garestier, T.; Helene, C. Reflectance and luminescence studies of molecular complex formation between tryptophan and nucleic acid components in frozen aqueous solutions. Biochemistry 1971, 10, 300-309.

55. Rajeswari, M.R.; Montenay-Garestier, T.; Helene, C. Does tryptophan intercalate in DNA? A comparative study of peptide binding to alternating and non-alternating A.cntdot.T sequences. Biochemistry 1987, 26, 6825-6831, [http:/ / dx.doi.org/10.1021/bi00395a036]. PMID: 3427045, doi:10.1021/bi00395a036.

56. Gates, D.M. Biophysical Ecology; Springer-Verlag, 1980.

57. Ulanowicz, R.; Hannon, B. Life and the production of entropy. Proc R Soc Lond B 1987, 232, $181-192$.

58. Schneider, E.D.; Kay, J.J. Complexity and thermodynamics: towards a new ecology. Futures 1994, 24, 626-647.

59. Kleidon, A. Entropy Production by Evapotranspiration and its Geographic Variation. Soil \& Water Res. 2008, 3, S89-S94.

60. Zotin, A.I., Bioenergetic trends of evolutionary progress of organisms. In Thermodynamics and regulation of biological processes; Lamprecht, I.; Zotin, A.I., Eds.; De Gruyter, 1984; pp. 451-458.

61. Schwartzman, D.; Lineweaver, C.H. The hyperthermophilic origin of life revisited. Biochem. Soc. Transact. 2004, 32, 168-171.

62. Limaye, S.S.; Mogul, R.; Smith, D.J.; Ansari, A.H.; Słowik, G.P.; Vaishampayan, P. Venus' Spectral Signatures and the Potential for Life in the Clouds. Astrobiology 2018, 18, 1181-1198, [https:/ / doi.org/10.1089/ast.2017.1783]. PMID: 29600875, doi:10.1089/ast.2017.1783. 
63. Heinz, J.; Schulze-Makuch, D. Thiophenes on Mars: Biotic or Abiotic Origin? Astrobiology 2020, 20, 552-561, [https:/ / doi.org/10.1089/ast.2019.2139]. PMID: 32091933, doi:10.1089/ast.2019.2139.

64. Pershin, S. Correlation of "chlorophyll" and water index on mars surface. Microsymposium 36, MS079, 2002.

65. López-Puertas, M.; Dinelli, B.M.; Adriani, A.; Funke, B.; García-Comas, M.; Moriconi, M.L.; D’Aversa, E.; Boersma, C.; Allamandola, L.J. Large Abundances of Polycyclic Aromatic Hydrocarbons in Titan's Upper Atmosphere. The Astrophysical Journal 2013, 770, 132. 